\title{
EVALUACIÓN DE LA ACTIVIDAD ANTIOXIDANTE DE SEIS FRUTALES AMAZÓNICOS: ANONA, CASTAÑA, CHOPE, HUASAÍ, HUITO Y UVILLA
}

\author{
Víctor SOTERO', Luz SILVA', Claudia MERINO-ZEGARRA', Martha MACO', Ericka DÁVILA², Wacner \\ RAMIREZ $^{2}$, Dora GARCIA ${ }^{2}$ \\ 1 Instituto de Investigaciones de la Amazonía Peruana (IIAP). Laboratorio de Sustancias Naturales Bioactivas (LSNB). Carretera \\ Iquitos-Nauta, km 4,5. Iquitos, Perú. e-mail:vsotero@iiap.org.pe \\ 2 Universidad Nacional de la Amazonía Peruana (UNAP). Facultad de Ingeniería Química. Departamento de Química. Freyre 616. \\ Iquitos, Perú.
}

\section{RESUMEN}

En el presente estudio se realizó la evaluación de la actividad antioxidante mediante el secuestro de radicales libres del DPPH de los frutos de anona, castaña, chope, huasaí, huito y uvilla, frutos originarios de la cuenca amazónica. Así mismo, se determinó la concentración de compuestos fenólicos por espectrofotometría y ácido ascórbico por cromatografía de HPLC. La mayor actividad antioxidante se da en las cáscaras del chopé con $\mathrm{IC}_{50}$ de $63.02 \mathrm{ug} / \mathrm{ml}$. En cuanto a compuestos polifenólicos, presentó mayor concentración la cáscara y pulpa de huito 137.15 y 97.78 $\mathrm{mg} / 100 \mathrm{~g}$ respectivamente. En tanto que en ácido ascórbico están presentes en altas concentraciones en la pulpa de anona con $4.28 \mathrm{mg} / 100 \mathrm{~g}$ y semilla de castaña con $3.33 \mathrm{mg} / 100 \mathrm{~g}$.

PALABRAS CLAVE: frutos amazónicos, antioxidantes, ácido ascórbico.

\section{EVALUATION OF ANTIOXIDANT ACTIVITY OF SIX AMAZONIAN FRUITS: ANONA, BRAZIL NUT, CHOPE, ACAI BERRY, HUITO AND UVILLA.}

\begin{abstract}
The antioxidant activity of six Amazonian fruits (anona, brazil nut, chope, acai berry and uvilla) were evaluated by measuring the sequestration of free radicals in DPPH. Additionally, the concentration of phenolic compounds was measured using a spectrophotometer and ascorbic acid was measured using chromatography of HPLC. The peel of chopé has the highest antioxidant activity with $\mathrm{IC}_{50}$ of $63.02 \mathrm{ug} / \mathrm{ml}$. The highest concentration of phenolics was found in huito, both in the peel and in the pulp (137.15 and $97.78 \mathrm{mg} / 100 \mathrm{~g}$, respectively.) The highest ascorbic acid concentrations were in anona $(4.28 \mathrm{mg} / 100 \mathrm{~g})$ and brazil nut $(3.33 \mathrm{mg} / 100 \mathrm{~g})$.
\end{abstract}

KEYWORDS: Amazonian fruits, antioxidants, ascorbic acid. 


\section{INTRODUCCIÓN}

Las especies conocidas como anona (Rollinia mucosa (Jacq.) Baillón), castaña (Bertholletia excelsa H.B.K), huito (Genipa americana), uvilla (Pouroma cecropiafolia Mart.), chopé (Gustavia longifolia) y huasaí (Euterpe precatoria), son nativas del bosque amazónico y sus frutos son de apreciable valor alimenticio y forman parte de la dieta del poblador amazónico (Villachica, 1996; Flores, 1997; Henderson et al., 1995).

Los antioxidantes, son sustancias que permiten proteger a las moléculas de las células del daño oxidativo producido por las especies reactivas del oxígeno (ROS), como el radical hidroxilo $\left(\mathrm{OH}^{\circ}\right)$, superóxido $\left(\mathrm{O}_{2}{ }^{-}{ }^{-}\right)$, peroxilo $\left(\mathrm{Ro}_{2}{ }^{\circ}\right)$ u óxidos de nitrógeno $\left(\mathrm{NO}^{\circ}, \mathrm{NO}_{2}{ }^{\circ}\right)$. Las células cuentan con mecanismos de protección, pero cuando este sistema falla, se produce un desequilibrio y se produce la generación de especies oxidantes produciendo el estrés oxidativo, proceso donde los ROS atacan los lípidos, inactivan proteínas o dañan el ADN. Los principales antioxidantes naturales son el acido ascórbico, el -tocoferol, los carotenoides y los compuestos fenólicos, los cuales son encontrados en diferentes fuentes vegetales. Estos antioxidantes actúan reaccionando con los radicales libres, Y deteniendo la reacción de propagación en cadena. $\mathrm{Su}$ forma de actuación puede representarse esquemáticamente:

$$
\mathrm{R}^{\bullet}+\mathrm{AH} \longrightarrow \mathrm{RH}+\mathrm{A}^{\bullet}
$$

Donde ka especie reactiva del oxigeno $\mathrm{R}^{\circ}$, se transforma en una molécula y el antioxidante $\mathrm{AH}$ se transforma en el radical libre $\mathrm{A}^{\circ}$. La diferencia fundamental es que el radical libre del antioxidante no es lo suficientemente reactivo para seguir dando lugar a reacciones de propagación, y se destruye o une con otro radical libre, dando entonces una molécula estable (Aruoma, 1993; Gutteridge \&Halliwell,1994).

Los antioxidantes pueden ser sintéticos o naturales, los primeros se utilizan ampliamente para proteger ciertos alimentos, pero sus posibles efectos secundarios limitan su utilización, por eso es que las investigaciones se han orientado a los productos naturales. Los antioxidantes naturales son encontrados en diversas partes de las plantas, como semillas, frutos, hojas, corteza y la raíz, en los productos microbianos de fermentación y de reacción de Maillard entre otros. Las principales fuentes de antioxidantes, fueron inicialmente investigadas en las plantas utilziads como especias y condimentos (Chipault et al., 1952; Inataní et al., 1982; Nakatani \& Inatani, 1984; Namiki, 1990). En frutos amazónicos también se encuentran los trabajos de actividad antioxidante como os de Garcia et al. (2003, 2009,
2011), Sotero et al. $(2009,2011)$ y Gonzales \& Torres (2011).

La vitamina $\mathrm{C}$ o ácido ascórbico, presenta una estructura bastante sencilla, pues se trata de la lactona de un azúcar-ácido, el ácido ascórbico sólo se precisa en la dieta de unos pocos vertebrados, entre ellos el hombre. Algunos insectos y otros invertebrados necesitan también ácido ascórbico, pero la mayor parte de los demás animales superiores y de los vegetales pueden sintetizarlo a partir de la glucosa y de otros precursores sencillos. El ácido ascórbico es un potente reductor, pierde con facilidad átomos de hidrógeno y se transforma en ácido dehidroascórbico, que también posee actividad de vitamina C. Sin embargo, la actividad vitamínica se pierde cuando el anillo lactónico del ácido dehidroascórbico se hidroliza para rendir ácido dicetogulónico. El ácido ascórbico presente en los alimentos se destruye, en su mayor parte por cocción (Pirone et al., 2003; Lehninger, 1987).

Los compuestos fenólicos, presentan en su estructura uno o mas anillos aromáticos con al menos un sustituyente hidroxílico. Están ampliamente distribuidos en el reino vegetal, se metabolizan como metabolitos secundarios con funciones de defensa, y son por lo general responsables por el color, astringencia y sabor de los vegetales. Se les encuentra en las verduras y frutas y en productos elaborados como el vino y cerveza (Bravo, 1998; Wollgast \& Anklam, 2000; Dreosti, 2000).

Este trabajo ttuvo como objetivo evaluar la capacidad antioxidante de los frutos de anona, castaña, chopé, huasaí, huito y uvilla.

\section{MATERIALES Y MÉTODOS}

\section{MATERIAL BIOLÓGICO}

Los frutos de huasai y uvilla se colectaron en el Centro de Investigaciones "Allpahuayo" del Instituto de Investigaciones de la Amazonia Peruan (IIAP), la castaña en Tamshiyacu (Distrito de Sargento Lores) y la anona, el huito y el chope de los productores a lo largo de la carretera Iquitos - Nauta.

\section{DETERMINACIONES ANALÍTICAS}

En los análisis bromatológicos, se obtuvo humedad, proteínas, grasas y minerales de acuerdo a lo indicado por Osborne \& Vgogt (1986). La concentración de carbohidratos se realizó por diferencia de peso. En cuanto a la actividad antioxidante, se obtuvieron extractos metanólicos de muestras secas, se evaluó la actividad antioxidante mediante el método de Lebeu $e t$ al. (2000), por reducción del radical 1.1-difenil-2picrilhidrazil (DPPH), con absorbancia de $515 \mathrm{~nm}$. La 
determinación de polifenoles se realizó según la técnica de Valls et al. (2000), utilizando el reactivo de Folin Ciocalteu y un patrón de catequina para la curva estándar, efectuándose la lectura a $765 \mathrm{~nm}$. Así mismo, se determinó la concentración del ácido ascórbico, siguiendo la metodología dada por Sotero et al. (2009), empleando la cromatografía de HPLC, equipado con detector de absorbancia, fase reversa 5 $\mu \mathrm{m}$. Con columna LiChrocart RP18 $(250 \times 4.6 \mathrm{~mm})$.

\section{RESULTADOS Y DISCUSIÓN}

De acuerdo a la Tabla 1, se observa las dimensiones de los frutos frescos de anona, Castaña, chope, huito, huasai y uvilla, destacando el fruto de la anona con $18.8 \times 15.6 \mathrm{~cm}$, y castaña con $4.64 \times 2.43 \mathrm{~cm}$. Se obtuvo mayor peso de pulpa en el de anona $(892 \mathrm{~g}$ ).

De acuerdo a los análisis bromatológicos de los frutos (Tabla 2), se observa alta concentración de grasa y proteínas en la semilla de castaña con $40.0 \mathrm{~g} / 100 \mathrm{~g}$ y $46.3 \mathrm{~g} / 100 \mathrm{~g}$, así como chope con $30.2 \mathrm{~g} / 100 \mathrm{~g}$ y 28.8 $\mathrm{g} / 100 \mathrm{~g}$, en pulpa seca, respectivamente.

El resumen de los porcentajes de inhibición de los radicales producidos por el DPPH de los frutos en estudio en extractos metanólicos tanto en pulpa, como en cáscara y semilla (Tabla 3 ), se observa lo siguiente:

El $\mathrm{IC}_{50}$ en pulpas, de menor a mayor se encuentran en las pulpas de huasai (mezclada con la cáscara debido a su dificultad de separarlas), chopé y huito las que reportan $1347.94, \quad 5715.44$ y $8261.01 \mathrm{ug} / \mathrm{ml}$ respectivamente. $\mathrm{El} \mathrm{IC}_{50}$ de las pulpas es similar a los reportados por Gonzales \& Torres (2011), quienes reportan para anihuayo (Plinia clausa) con 2810.0 $\mathrm{mg} / \mathrm{ml}$, ungurahui (Oenocarpus bataua) con 2140.0 $\mathrm{mg} / \mathrm{ml}$, macambillo (Theobroma subincanum) con $5910.0 \mathrm{mg} / \mathrm{ml}$ y ubos (Spondias bombin) con 160.0 $\mathrm{mg} / \mathrm{ml}$.
La mayor actividad antioxidante en cáscaras presentó el chopé, que presenta un $\mathrm{IC}_{50}$ de $63.02 \mathrm{ug} / \mathrm{ml}$. Las cáscaras de anona y uvilla mejoran notablemente su actividad a $1000 \mathrm{ug} / \mathrm{ml}$, alcanzando $96.25 \%, 96.37 \%$ y $96.37 \%$ de inhibición respectivamente.

Entre las semillas destacan las de uvilla y huasai que alcanzan un $\mathrm{IC}_{50}$ de 1544.59 y $9449.31 \mathrm{ug} / \mathrm{ml}$. Las semillas de castaña y chope tienen una baja actividad antioxidante, comparando los $\mathrm{IC}_{50}$ de las muestras estudiadas, se observa que tienen una actividad antioxidante moderada, si comparamos con otras especies amazónicas como semillas de cacao (Theobroma cacao), cacahuillo (Herrenia nitida), copoazu (Theobroma grandiflorum) y macambo (Theobroma bicolor) con 520.0, 169.0, 232.0 y 755.0 $\mathrm{ug} / \mathrm{ml}$ respectivamente (Sotero et al., 2011).

De acuerdo a la Tabla 4, se observa que la mayor concentración de compuestos fenólicos se encuentra en pulpa de huito $(137.15 \mathrm{mg} / 100 \mathrm{~g})$, en la mezcla pulpa/cáscara de huasai $(56.799 \mathrm{mg} / 100 \mathrm{~g})$ y en la semilla de uvilla $(66.401 \mathrm{mg} / 100 \mathrm{~g})$, guardando una relación con actividad antioxidante de esta parte de las muestras evaluadas líneas arriba.

La concentración de ácido ascórbico en las pulpas de los frutos en estudio, se presentan en la Tabla 5. Se puede observar que el aporte de estos es bajo, destacando ligeramente la pulpa de anona con 4.28 $\mathrm{mg} / 100 \mathrm{~g}$, y castaña con $3.33 \mathrm{mg} / 100 \mathrm{~g}$. Cabe mencionar que otras especies amazónicas también presentan concentraciones similares, como es el caso de anihuayo (Plinia clausa), ungurahui (Oenocarpus bataua), macambillo (Theobroma subincanum) y ubos (Spondias bombin) con 7.27, 0.81, 5.62 y 3.54 $\mathrm{mg} / 100 \mathrm{~g}$ respectivamente (Gonzáles \& Torres, 2011), muy diferente al camu camu (Myrciaria dubia H.B.K.) que presenta hasta $2880.0 \mathrm{mg} / 100 \mathrm{~g}$ (Flores, 1997). 
FOLIA

Tabla 1. Dimensiones de los frutos frescos de anona, castaña, chope, huito, huasai y uvilla.

\begin{tabular}{lccccc}
\hline \multicolumn{1}{c}{ VARIABLE } & ANONA & CASTAÑA & CHOPE & HUITO & UVILLA \\
\hline Diámetro longitudinal $(\mathrm{cm})$ & $18.8 \pm 2.5$ & $4.64 \pm 0.49$ & $4.74 \pm 0.34$ & $7.45 \pm 0.76$ & $2.4 \pm 0.31$ \\
Diámetro transversal $(\mathrm{cm})$ & $15.6 \pm 0.9$ & $2.43 \pm 0.30$ & $5.87 \pm 0.51$ & $6.17 \pm 0.26$ & $1.3 \pm 012$ \\
Peso $(\mathrm{g})$ & $1600 \pm 12.9$ & $612.0 \pm 130.65$ & $93.71 \pm 23.09$ & $157.0 \pm 20.34$ & $13.7 \pm 1.8$ \\
Peso pulpa $(\mathrm{g})$ & $892 \pm 9.23$ & - & $20.1 \pm 6.67$ & $98.63 \pm 16.6$ & $7.05 \pm 0.28$ \\
Peso cáscara de fruto $(\mathrm{g})$ & $548 \pm 25.2$ & $521 \pm 12.0$ & $39.6 \pm 8.75$ & $17.04 \pm 5.7$ & $2.0 \pm 0.5$ \\
Peso semilla $(\mathrm{g})$ & $60 \pm 2.3$ & $8.2 \pm 1.17$ & $29.3 \pm 8.03$ & $33.28 \pm 8.68$ & $83.0 \pm 0.58$ \\
Peso pulpa de semilla $(\mathrm{g})$ & - & $3.6 \pm 0.60$ & - & - & - \\
Peso cáscara de semilla $(\mathrm{g})$ & - & $4.6 \pm 0.5$ & - & - & - \\
\hline
\end{tabular}

Tabla 2. Análisis bromatológico (g/100g) en peso seco de pulpa de los frutos de anona, castaña, chope, huasaí, huito y uvilla.

\begin{tabular}{lccccc}
\hline \multirow{2}{*}{ FRUTO } & \multirow{2}{*}{ HUMEDAD } & \multicolumn{4}{c}{ PESO SECO $(\mathbf{g} / \mathbf{1 0 0 g}$.) } \\
\cline { 3 - 6 } & & PROTÉ́NA & GRASA & CENIZA & CARBOHIDRATOS* \\
\hline Anona & $81.8 \pm 0.006$ & $14.4 \pm 0.2$ & $1.0 \pm 0.01$ & $3.6 \pm 0.05$ & 81.0 \\
Castaña & $14.8 \pm 1.21$ & $46.3 \pm 3.5$ & $40.0 \pm 0.2$ & $3.9 \pm 1.14$ & 9.8 \\
Chope & $49.7 \pm 2.70$ & $28.8 \pm 6.3$ & $30.2 \pm 0.5$ & $2.1 \pm 0.023$ & 38.9 \\
Huasai & $42.8 \pm 10.17$ & $8.9 \pm 1.0$ & $18.5 \pm 0.6$ & $1.5 \pm 0.02$ & 71.3 \\
Huito & $81.3 \pm 0.001$ & $15.1 \pm 2.3$ & $11.1 \pm 0.1$ & $3.8 \pm 0.1$ & 70.9 \\
Uvilla & $84.9 \pm 1.69$ & $2.9 \pm 0.9$ & $0.1 \pm 0.1$ & $0.2 \pm 0.001$ & 96.8 \\
\hline
\end{tabular}


Tabla 3. Determinación de la actividad antioxidante de los extractos metanólicos de seis frutos amazónicos por reducción del radical libre del DPPH.

\begin{tabular}{lcccccc}
\hline \multicolumn{1}{c}{ FRUTO } & \multicolumn{7}{c}{ EXTRACTOS METANÓLICOS (ug/ml) } \\
\hline Pulpa & $\mathbf{1 0 0}$ & $\mathbf{3 0 0}$ & $\mathbf{1 0 0 0}$ & $\mathbf{3 0 0 0}$ & $\mathbf{1 0 0 0 0}$ & IC $_{50}$ \\
\hline Anona & 5.6 & 5.16 & 19.09 & - & - & - \\
Chope & 3.71 & 7.66 & 13.67 & 32.10 & 78.26 & $5715.44 \pm 0.089$ \\
Huasai & 14.74 & 19.33 & 45.68 & 70.23 & 87.87 & $1347.94 \pm 0.085$ \\
$\begin{array}{l}\text { (pulpa/cáscara) } \\
\text { Huito }\end{array}$ & 11.6 & 8.36 & 12.93 & 23.64 & 59.13 & $8261.01 \pm 0.019$ \\
Uvilla & 11.38 & 10.38 & 21.55 & - & - & - \\
\hline Cáscara & & & & & & \\
Anona & 6.33 & 13.01 & 22.63 & 35.88 & 83.68 & $5066.66 \pm 0.105$ \\
Chope & 73.30 & 96.36 & 96.00 & & & $63.02 \pm 0.004$ \\
Huito & 10.98 & 12.19 & - & - & - & - \\
Uvilla & 12.95 & 15.36 & 96.37 & - & & - \\
\hline Semilla & & & & & & \\
Anona & 6.86 & 13.01 & 13.32 & 35.88 & 51.22 & $9449.31 \pm 0.14$ \\
Castaña & 14.31 & 15.72 & 21.55 & - & - & - \\
Chope & 5.95 & 6.30 & 15.98 & - & - & - \\
Huasai & 13.13 & 18.46 & 96.37 & 68.25 & 90.89 & $1544.59 \pm 0.02$ \\
Uvilla & $53-98$ & 74.12 & 87.65 & - & - & $700.47 \pm 0.041$ \\
\hline
\end{tabular}

Tabla 4. Polifenoles totales $(\mathrm{mg} / 100 \mathrm{~g})$ en frutos, cáscara y semilla de frutales amazónicos.

\begin{tabular}{lcccccc}
\hline $\begin{array}{c}\text { PARTE } \\
\text { DEL FRUTO }\end{array}$ & ANONA & CASTAÑA & CHOPE & HUASAI & HUITO & \multirow{2}{*}{ UVILLA } \\
\hline Pulpa & $20.98 \pm 0.02$ & - & $26.71 \pm 0.01$ & $56.80 \pm 0.25^{*}$ & $137.15 \pm 0.03$ & $12.72 \pm 052$ \\
Cáscara & $32.22 \pm 0.03$ & - & $66.40 \pm 06$ & - & $7.92 \pm 0.05$ & $35.35 \pm 0.26$ \\
Semilla & $23.68 \pm 0.02$ & $1.48 \pm 0.04$ & $49.75 \pm 0.03$ & $31.65 \pm 0.08$ & $26.81 \pm 0.017$ & $66.40 \pm 0.05$ \\
\hline
\end{tabular}

*Mezcla de pulpa con cáscara.

Tabla 5. Concentración de ácido ascórbico en pulpa de seis frutos amazónicos.

\begin{tabular}{lc}
\hline MUESTRAS & $\begin{array}{c}\text { ÁCIDO ASCÓRBICO } \\
\text { (mg/100g) }\end{array}$ \\
\hline Chope & $1.62 \pm 0.09$ \\
Anona & $4.28 \pm 0.08$ \\
Huasaí & $2.60 \pm 0.17$ \\
Huito & $1.77 \pm 0.05$ \\
Castaña & $3.33 \pm 0.06$ \\
Uvilla & $2.45 \pm 0.20$ \\
\hline
\end{tabular}




\section{BIBLIOGRAFÍA CITADA}

Bravo, L. 1998. Chemistry dietary sources metabolism and nutritional significancence Nutr. Rev. Vol. 56(11)317-333.

Chipault, J. R.; Mizuno, G. R.; Lundberg, W. O. 1952. The antioxidant properties of natural spices. Food Res. 17:46-55.

Dreosti, J. E. 2000. Antioxidant polyphenols in tea, cocoa and wine. Nutrition. Rev. Vol. 16 (7/8):692694.

Flores, S. 1997. Cultivo de frutales natives amazonicos. Manual para el extensionista. Tratado de Cooperación amazónica. Secretaria ProTempore. Lima. Ed. Mirigraf. 307p.

García, G.; Pavan Torres, R.; Mancini, D.; Mancini Filho, J. 2003. Actividad Antioxidante de los frutos de la Sacha Mangua (Grias neubertii Macbr.). Rv. Alimentaria, Madrid, 343:87-91.

García, D.; Sotero, V.; Mancini, G.; PavanTorres, R. 2011 Evaluación de la actividad antioxidante in vivo de tres frutos de la amazonia: G. augusta L, Grias neubertii Macbr y Theobroma bicolor. Rev. Soc. Quím. Perú. Vol. 77(1): 44-55. 2011.

Garcia, G.; Sotero,V.; Mancini, D.; Torres, R. P.; Mancini filho, J. 2009. Actividad antioxidante de los extractos del chope (Gustavia augusta L). Rev. Soc. Quim. Peru, Vol.(75)3:374-381.

Gonzales, A.; Torres G. 2011. Contribuciones al conocimiento de frutales nativos amazónicos. Iquitos.Editora: Perucuadros EIRL, $136 \mathrm{p}$.

Gutteridge, J. M. C.; Halliwell, B. 1994. Antioxidants in nutrition, health and disease. Oxford. New York: Oxford Universit. 143p.

Henderson, A.; Galeano, G.; Bernal, R. 1995. Field Guide to the palms of the Americas. Princeton University Press. Princeton. New Jersey. 124p.

Inatani, R.; Nakatani, N.; Seto, H. 1982. Structure of a new antioxidative phenolic diterpene isolated from rosemary (Rosmarinus officinalis L.). Agric. Biol. Chem. 46:1661-63.
Lehninger, A. 1987. Bioquímica. Las bases moleculares de la estructura y función celular. Barcelona. Ed. Omega. 357-358 pp.

Nakatani, N.; Inatani, R. 1990. Two antioxidative diterpenes from rosemary (Rosmarinus officinalis L.) revised structure for rosmanol. Agric. Biol. Chem. 48:2081-83

Namiki, M. 1990. Antioxidants/antimutagenics in food. Crit. Rev. Food Sci. Nutr. Vol. 29 (4): 273-300.

Osborne, D. R.; Vgogt, P. 1986. Análisis de los nutrientes de los alimentos. Zaragoza. Ed. Acribia S.A. 258 p.

Pirone, B. N.; Ochoa, M. R.; Kesseler, A. G.; De Michelis, A. 2003. Evolución de la concentración de ácido ascórbico durante el proceso de deshidratación de frutos de la rosa mosqueta (Rosa eglanteria L.). Revista de Investigaciones Agropecuarias. Vol 31(1):85-98.

Sotero, V.; Silva, L.; Garcia, D.; Iman, S. 2009. Evaluación de la actividad antioxidante de la pulpa, cáscar y semilla del fruto del camu camu. Rev. Soc. Quim. Peru. Vol. (75) 3:293-299.

Sotero, V.; Maco, M.; Vela, J.; Merino, C.; Dávila, E.; García, D. 2011. Evaluación de la actividad antioxidante y compuestos fenólicos en pulpa y semillas de cuatro frutales amazónicos de la familia Sterculiaceae. Rev. Soc. Quím. Perú. Vol. 77 (1): 66-74.

Valls, J.; Lampreave, M.; Nadal, M.; Arola, L. 2000. Importancia de los compuestos fenólicos en la calidad de los vinos tintos de crianza. Alimentación, pp 119-124.

Wollgast, J.; Anklam, E. 2000. Review on polyphenols in Theobroma cacao: changes in composition during the manufacture of chocolate and methodology for identification and quantification. Food Res. Int. 33:423-427. 